\title{
Sozialpolitik: Vorwärts- und Rückwärtsreformen und Neuvermessung von Solidarität
}

Christine Trampusch

Die Agenda 2010 gilt - insbesondere mit den Hartz-Gesetzen - gemeinhin als Zäsur in der bundesdeutschen Sozialpolitik. Doch nicht nur der konsequente Rückbau bis dato verbriefter sozialstaatlicher Leistungen wird den Reformen attestiert, sondern auch die Aufweichung des gesellschaftlichen Solidaritätsgedankens. Der Beitrag hinterfragt solche zugespitzten Einschätzungen. Er macht zum einen deutlich, dass die gelockerte Reformbremse eher eine Pendelbewegung als eine Schubwirkung ausgelöst hat. Zum anderen wird aufgezeigt, wie gesellschaftliche Solidarität derzeit auf neue soziale Grundlagen gestellt wird.

\section{Einleitung}

Analysen zum gesellschaftlichen Wandel sollten stets auf einem längeren Betrachtungszeitraum fußen. Der Rückblick auf vergangene Entwicklungen ist allemal sinnvoll, um Epochen voneinander abzugrenzen. Ferner kann er dazu dienen, institutionelle Veränderungen, die ein Politikfeld wie die Sozialpolitik nachhaltig prägen und neu justieren, von Prozessen zu unterscheiden, die kurzfristigen Kalkülen und Konjunkturen der Politik entspringen. Erst die längerfristige Analyse ermöglicht, Kontinuität und Wandel zu identifizieren. Und sie reduziert die Gefahr, sich in Diskussionen zu verheddern, ob das Neue wirklich neu ist oder ob das Alte vielmehr runderneuert wurde.

Betrachtet man die deutsche Sozialpolitik über einen längerfristigen Zeitraum hinweg, so zeigen sich fundamentale Strukturveränderungen. Sie betreffen vor allem das Verhältnis zwischen Parteien- und Verbändesystem. Die mit der Agenda 2010 und den Hartz-Gesetzen losgetretenen Reformen sind dafür Ausdruck und Indiz. Die Sozialpolitik in „Post-Hartz Germany“ unterscheidet sich von der der Kohl-Ära in vielerlei Hinsicht: auf der Ebene der Entscheidungsstrukturen, auf der Ebene der Akteure und auch auf der Ebene der Maßnahmen. Ein wesentliches gemeinsames Merkmal der Verschiebungen auf allen drei Ebenen ist: Der Staat ist aktiver geworden. Er greift in die sozialstaatlichen Selbstverwaltungsstrukturen massiv ein (Reform der Bundesagentur für Arbeit (kurz: BAReform); Gesundheitsfonds)). Parteipolitische Opportunitäten schlagen sich zunehmend in der sozialpolitischen Agenda nieder (Verlängerung der Bezugsdauer des Arbeitslosengeldes I (kurz: ALG I)). Mehr noch: Die Eingriffe des Staates sind zum Teil kurzfristigen partei- und wahlpolitischen Kalkülen unterworfen: Da wird zunächst die Rente mit 67 eingeführt und dann im Folgejahr eine Rentenerhöhung verabschiedet, die die Riester-Reform auf den Kopf stellt. Doch auch das Verbändesystem ist heute viel fragmentierter und polarisierter als Mitte der 1990er Jahre, weil kommerzielle Anbieter von Sozialpolitik wie auch die Sozialverbände ihre Position stärken konnten, während die traditionell dominanten Akteure, sprich Arbeitgeber und Gewerkschaften, einem Fragmentierungs- und Pluralisierungsprozess unterliegen. Und außerdem: Neue Akteure stellen neue Forderungen an die Sozialpolitik. Sie soll neue Funktionen und Aufgaben erfüllen. Doch trotz dieser Dynamik gibt es nach wie vor Kontinuität im deutschen Sozialstaat. Von US-amerikanischen Verhältnissen sind wir weit entfernt. In den großen Parteien gibt es immer noch Kräfte, die sich einer bloß liberalen und restriktiven Sozialpolitik widersetzen.

Ich werde im Folgenden nicht nur auf die skizzierten institutionellen Veränderungen eingehen, sondern, der Themensetzung dieses Jubiläumsheftes folgend, gesamtgesellschaftliche Auswirkungen und Veränderungen ausleuchten. Dabei unterscheide ich zwischen politischen Folgen und gesellschaftlichen Folgen. Meine Thesen lauten:

(1) Die Neustrukturierung des Politikfeldes unterwirft die Sozialpolitik den Gesetzmäßigkeiten politischer Macht und politischen Wettbewerbs. Dies hat unter der Großen Koalition bereits zu einer Kadenz aus Vorwärts- und Rückwärtsreformen geführt.
(2) Es kommt zu einer „Neuvermessung von

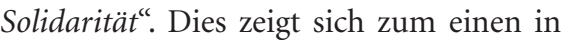
der Diskussion über Solidarität zwischen Jung und Alt, die jüngst virulent wurde, zum anderen im Umschwenken der Gewerkschaften hin zu einer tariflich gebundenen Solidarität.

\section{Celoste Reformbremse: \\ Gelöste Reformbremse: Riester, Agenda 2010, Hartz}

Die Riestersche Rentenreform 2001 sowie die im Zuge der Agenda 2010 durchgeführten Hartz-Reformen haben die Reformbremse im Politikfeld Sozialpolitik gelöst. Sie haben den deutschen Sozialstaat nachhaltig verändert. Nicht nur die Rentenpolitik, sondern auch die Arbeitsmarktpolitik - darin ist sich die Literatur weitgehend einig - hat mit diesen Reformen einen „Kurswechsel“ (Schmidt 2007, S. 304) erfahren (Lamping/Rüb 2004; Schmidt 2005; Streeck/Trampusch 2005; Trampusch 2006a). Manfred G. Schmidt umschrieb die Agenda 2010 auf eindrückliche Weise folgendermaßen: „Die Botschaft an die Bürger lautete: Ende der Ausbaustrecke der Sozialpolitik - Beginn einer Umbauund Rückbauzone - Weiterfahrt bereichsweise auf eigene Gefahr. Mit freundlichen

Christine Trampusch, Prof. Dr., Assistenz professorin für Vergleichende Politik am Institut für Politikwissenschaft der Universität Bern. Arbeitsschwerpunkte: Vergleichende Politik, Verbändeforschung, Europaforschung. e-mail: christine.trampusch@ipw.unibe.ch 
Grüßen, Ihre SPD-geführte Bundesregierung." (Schmidt 2007, S. 298). Wolfgang Streeck (2005, S. 2) nimmt neue Eliten in der Bundesrepublik wahr, die zu post-korporatistischen Politikformen führen und das „System horizontaler Elitenverflechtung" ablösen, das für das Nachkriegsmodell des Wohlfahrtskorporatismus „struktur- und stilbildend" war. Der Wohlfahrtskorporatismus ist in der Tat passé. In der Rentenpolitik wurde mit der Sanktionierung der privaten, betrieblichen und tariflichen Altersvorsorge die staatliche Verantwortung zugunsten der individuellen Verantwortung zurückgestellt. Die Hartz-Reformen beschnitten nicht nur den Einfluss der Verbände auf die Bundesagentur für Arbeit erheblich. Hartz IV, also die Zusammenlegung von Sozial- und Arbeitslosenhilfe auf Sozialhilfeniveau, hat auch die umfangreichste Leistungskürzung seit Bestehen der Bundesrepublik implementiert.

Der sozialpolitische Kurswechsel, den die rot-grüne Regierung zwischen 2001 und 2003 vollzogen hat, passte so gar nicht zum bis dahin vorherrschenden Bild einer sozialpolitischen Reformblockade, die das Land lähmte. Vor Riester und Hartz galt die Sozialpolitik im deutschen Regierungssystem als Paradebeispiel eines sektoralisierten und von Verbänden dominierten Politikfeldes. Belege für diese Einschätzung lieferte die Policy-Forschung: Sie hat nicht nur in der Renten-, Arbeitsmarkt- und Gesundheitspolitik institutionell verfestigte Verhandlungsnetzwerke zwischen Parteien und Verbänden identifiziert, sondern auch gezeigt, dass die Sozialpolitiker in den Parteien und Fraktionen - insbesondere in den 1970er und 1980er Jahren - mächtige Akteure waren, an denen Partei- und Fraktionsführungen sich auszurichten hatten (Winter 1997; Lehmbruch 2000). Dieses spezifische Geflecht - also das Vorhandensein gewachsener und sektoralisierter Beziehungsstrukturen der parteidemokratischen und staatlichen Akteure mit den Gewerkschaften und Arbeitgeberverbänden zählt in der wissenschaftlichen Diskussion zu den Charakteristika des deutschen Modells. So kennzeichnete Peter Katzenstein (1987, S. 364) in seiner Studie über den semisouveränen deutschen Staat die Sozialpolitik mit dem Begriff der „Depolitisierung", womit er darauf verwies, dass der Staat Aufgaben und Funktionen an die Sozialversicherungsträger, die damalige Bundesanstalt für Arbeit und die Wohlfahrtsverbände ,delegiert“ hatte (ebd.).Verände- rungen in den sozial- und arbeitsmarktpolitischen Regelungen wurden im vorparlamentarischen Raum von den Gewerkschaften und Arbeitgeberverbänden oftmals vorstrukturiert; in der Regierung war die Sozial- und Arbeitsmarktpolitik an den zuständigen Minister delegiert, im Parlament an die Sozialpolitiker von CDU/CSU und SPD, die mit den Verbänden feste Bindungen aufwiesen. Auch bei der Bewältigung der deutschen Wiedervereinigung wirkten diese Verhandlungsnetzwerke zwischen Sozialpolitikern und Sozialpartnern. Bundeskanzler Helmut Kohl gewährte seinem Arbeitsminister Norbert Blüm in der Gestaltung der sozial- und arbeitsmarktpolitischen Maßnahmen in den neuen Ländern nahezu freie Hand. Kohl akzeptierte Gewerkschaften und Arbeitgeberverbände als tragende Pfeiler dieses Prozesses, indem er sie in wichtige Entscheidungen einband. Der frühere BDI-Präsident Hans-Olaf Henkel nannte dies „Konsenssoße“. Manfred G. Schmidt verwies auf das Vorhandensein von „zwei Sozialstaatsparteien“ und eines „sozialstaatsfreundliche[n] Parteiensystem[s]“ (Schmidt 2005, S. 170), weil sich sowohl CDU/CSU als auch SPD ,dem Anliegen des Schutzes gegen Not, der Hilfe für Schwächere und der Eindämmung krasser gesellschaftlicher Unterschiede verschrieben" (ebd.) hatten.

Was aber sind die Ursachen dafür, dass die bisherige Reformbremse im neuen Jahrtausend so abrupt gelöst werden konnte? Die wissenschaftliche Literatur bietet unterschiedliche Antworten: Schmidt (2007, S. 305) führt eine „komplizierte Mischung aus schweren ökonomischen, gesellschaftlichen und finanzpolitischen Problemen" an. Nullmeier (2002) fragt, ob wir auf dem Weg zu „Wohlfahrtsmärkten“ sind. Streeck (2008) geht viel weiter. Er vermutet hinter der Transformation der Sozialpolitik eine Transformation des deutschen Kapitalismus. In meinen eigenen Analysen (Trampusch 2006a, 2006b) habe ich den Wandel als eine Tendenz hin zum „Postkorporatismus" (Trampusch 2006b) bezeichnet und innenpolitische Verschiebungen im Parteien- und Verbändesystem als Ursache des Wandels identifiziert. Zu diesen gehören:

(1) Der Elitenwechsel in der Sozialpolitik, also der Übergang von Sozialpolitikern zu Berufspolitikern und die zunehmende Trennung der Sozialpolitiker von den sozialpolitischen Verbänden, womit das Parteiensystem ein höheres Maß an Autono- mie gegenüber dem Verbändesystem gewonnen hat.

(2) Die Fragmentierung der Gewerkschaften und Arbeitgeberverbände, weil bei beiden die innerverbandliche Solidarität und Loyalität mit dem Sozialstaats- und Tarifpolitikmodell der alten Bundesrepublik im Zuge verstärkter wirtschaftlicher Internationalisierung und sich ausdifferenzierender Arbeitnehmerinteressen abgenommen haben.

(3) Neue sektorale Wettbewerber wie privatgewerbliche Dienstleistungsunternehmen und Sozialverbände, die mittlerweile mit den Sozialpartnern konkurrieren.

(4) Der Strukurwandel, den die Selbstverwaltung vollzieht. Dies geschieht einerseits aufgrund des bereits in den 1970er Jahren eintretenden Trends ihrer „Entfunktionalisierung durch den Gesetzgeber" (Standfest 1977, S. 429), andererseits auch wegen ihrer Reorganisation durch den Staat.

(5) Die Marktstrukturen, die mittlerweile in allen Bereichen der Sozialpolitik eingeführt worden sind.

Dies alles hat die Beschränkung der politischen Macht durch korporatistische Strukturen aufgehoben. Mit der Rentenreform 2001, der Agenda 2010 und den HartzReformen ist der Staat aktiv geworden. Die Parteien haben deutlich an verbandsunabhängiger Politikformulierungskompetenz hinzugewonnen. Damit hat die Transformation der Sozialpolitik aber nicht nur mehr Parteienwettbewerb zur Folge, sondern auch - wie die folgende Analyse der Sozialpolitik der Großen Koalition zeigt eine gewisse Volatilität der Reformen.

\section{Kontinuität versus Richtungswechsel}

Nimmt man das Erbe der rot-grünen Regierung - die Sozialreformen Walter Riesters sowie die Agenda 2010 und die HartzKommission - zum Ausgangpunkt einer Bewertung der Sozialpolitik der Großen Koalition, so wird Gegenläufiges erkennbar. Auf der einen Seite hat die Große Koalition die von Schröder vorgegebene Linie fortgesetzt (Vorwärtsreformen); auf der 
anderen Seite hat sie diese jedoch auch korrigiert (Rückwärtsreformen). Daneben gab es mit der Pflegereform auch eine Leistungsausweitung. Die Pflegereform brachte höhere Leistungen, deren Dynamisierung und neue Betreuungsmöglichkeiten mit sich. Vermieden hat die Koalition jedoch eine Reform des Finanzierungssystems. Mit der von Finanzwissenschaftlern geforderten Umstellung auf ein kapitalgedecktes Verfahren wird sich erst die neue Regierung beschäftigen müssen.

\subsection{VORWÄRTSREFORMEN}

Was gehört zu den Vorwärtsreformen?

(1) Der in der Rentenpolitik bis Ende 2007 eingeschlagene Reformpfad wurde nicht verlassen. Die zwischen 2012 und 2029 schrittweise Einführung der Rente mit 67 sowie die Weiterführung der Beitrags- und Steuerfreiheit der Entgeltumwandlung auf Dauer (!) haben den mit Riester verbundenen Paradigmenwechsel eindeutig fortgeführt. Dabei hat Letzteres den von den Tarifpartnern eingeschlagenen Weg verstetigt, Aufgaben, die vormals in staatlicher Hand lagen, in eigene Regie zu übernehmen.

(2) Die Arbeitsmarktpolitik ließ die neue Koalition nach dem strengen Reformkurs der Vorgängerregierung erstmal unberührt; jedoch haben die Gerichte, allen voran das Bundessozialgericht und das Bundesverfassungsgericht, in aufsehenerregenden Urteilen der Politik mittlerweile Handlungsaufträge erteilt (für einen Überblick vgl. FAZ vom 29.02.2008, S. 14). Nichts anderes als politisches Tagesgeschäft sind die Reform der Selbstständigkeitsförderung (2006), die Schaffung des Saisonkurzarbeitergeldes (2006), die verschiedenen arbeitsmarktpolitischen Sonderprogramme für Problemgruppen sowie die Ersetzung des umstrittenen BA-Aussteuerungsbeitrages durch den BA-Eingliederungsbeitrag, mit dem sich die Bundesagentur für Arbeit (BA) nun zu $50 \%$ an den Kosten für Eingliederungsmaßnahmen und den Verwaltungskosten für ALG II-Bezieher beteiligen muss. Bemerkenswert ist jedoch die Senkung des Beitrages zur BA von 6,5\% auf $3,3 \%$, die zum einen durch die Sozialkürzungen der Vorgängerregierung und zum anderen durch die 2007 vollzogene Erhöhung der Mehrwertsteuer von $16 \%$ auf $19 \%$ finanziert wurde. Und: In der Ar- beitsmarktpolitik ist weiterer Wandel vorprogrammiert: Weil das Bundesverfassungsgericht (BVerfG) Ende Dezember 2007 die Arbeitsgemeinschaften zwischen BA und Kommunen für verfassungswidrig erklärt hat, muss die Regierung - egal welcher Coleur - bis Ende 2010 eine neue Lösung für einen der zentralen Pfeiler der Hartz-Reformen finden, nämlich die $\mathrm{Zu}-$ sammenlegung zwischen Sozial- und Arbeitsämtern. Möglicherweise trägt das Urteil sogar dazu bei, dass sich am Ende die seit Jahrzehnten diskutierte Option einer Kommunalisierung der Arbeitsmarktpolitik durchsetzt und damit das in einigen Kommunen praktizierte Optionsmodell, welches von Teilen der CDU/CSU und vor allem von den Landkreisen und ihrem Verband, dem Deutschen Landkreistag (DLT), aus institutionellen Eigeninteressen präferiert wird.

(3) Für die Gesundheitsreform mag zwar angesichts der schwierigen Kompromisslage zwischen CDU, CSU und SPD im Vorfeld dieser letztendlich 2007 verabschiedeten Reform - der schöne Helvetismus „Der Berg hat eine Maus geboren“ gelten. Das bringt jedoch Lösungen mit sich, die politischen Kompromissen in der Nähe eines kleinsten gemeinsamen Nenners geschuldet sind. Dennoch ist gerade der Gesundheitskompromiss nicht zu unterschätzen. Der Politisierungsgrad, der mit ihm bis heute einhergeht, indiziert, sich näher mit der Reform zu befassen. Der vom Bundesversicherungsamt verwaltete Gesundheitsfonds, welcher 2009 eingeführt werden soll und alle Beitragseinnahmen verwalten wird, hebt die seit 125 Jahren (seit 1883) bestehende Beitrags- und Finanzautonomie der Krankenkassen auf. Das ist allemal ein bemerkenswerter Bruch. Bereits 2008 soll der Beitragssatz vereinheitlicht werden. Der Staat positioniert sich in diesem Politikbereich, was auch daran deutlich wird, dass das Gesundheitsbudget in Zukunft mittels staatlicher Finanzzuweisungen verteilt werden soll. Entgegen der weitläufig vertretenen Meinung spricht dies jedoch nicht grundsätzlich gegen eine weitere Vermarktlichung der Gesundheitspolitik und die Stärkung von Wettbewerbselementen in diesem Politikbereich. Zwar kommt es durch die anvisierte Verstaatlichung der Ressourcen zunächst zu einer Verzerrung des Wettbewerbs; Liberalisierungsprozesse - darauf verweist die vergleichende politische Ökonomie - vollziehen sich jedoch in politisch hoch regulierten, korporatistischen Bereichen in der Regel in zwei Schritten: In einem ersten Schritt muss sich der Staat das Politikfeld von den Interessengruppen zurückerobern, mithin von den Gesetzlichen Krankenkassen, ihren Verbänden und den damit verwobenen Landesregierungen. Ist dies vollbracht, können in einem zweiten Schritt Reformen vollzogen werden, die den Marktkräften Platz machen.

\subsection{RÜCKWÄRTSREFORMEN}

Die Phase der Vorwärtsreformen wurde bereits im Herbst 2006 konterkariert. Es waren jedoch anfänglich nicht die traditionellen Sozialdemokraten, die sich den Wirtschaftsliberalen der Koalition widersetzten. Das Aufbäumen ging von der CDU aus. Es entsprang dem Chor sozialpolitischer christdemokratischer Stimmen (vgl. dazu auch Clemens 2007). Stimmführer wurde alsbald der Ministerpräsident NordrheinWestfalens, Jürgen Rüttgers, dessen Bundesland und Landesverband traditionell den sozialpolitischen Flügel der CDU repräsentiert. Nachdem es Schröder in seiner zweiten Legislaturperiode gelungen war, die CDU/CSU rechts zu überholen, begannen nun Teile der CDU damit, links an der SPD vorbeizuziehen.

Der Streit um die Verlängerung der Arbeitslosengeldbezugsdauer illustriert diesen Umbruch im Reformkurs besonders deutlich. Diese Korrektur wurde vom neuen „Sozialrebell“ (Stuttgarter Zeitung vom 23.11.2006, S. 3) der CDU, Jürgen Rüttgers, bereits im Herbst 2004 im Vorfeld der Landtagswahlen in Nordrhein-Westfalen mit Blick auf die damaligen Landtagswahlniederlagen der CDU in Brandenburg und Sachsen lanciert (FR vom 23.09.2004, S. 4). Ende 2007 wurde sie schließlich von der Koalition nach dem Dresdner Parteitag der CDU (November 2006) verabschiedet. Allerdings sollte die Nachbesserung zum ALG I-Bezug für Ältere nicht überbewertet werden, selbst wenn sie den mit der Agenda 2010 vollzogenen Wandel konterkariert. Denn die verlängerte Bezugsdauer des Arbeitslosengeldes I steht heute in einem anderen sozialrechtlichen Kontext als noch Mitte der 1990er Jahre. Damals galten noch generöse rentenrechtliche Regelungen, die erlaubten, die verlängerte Bezugsdauer als Brücke in die Frühverrentung zu nutzen. Dieser Weg ist heute verbaut aufgrund der massiven Reformen im Frühverrentungs- 
regime, die bereits die Regierung Kohl 1996 eingeleitet hatte.

Während die CDU - wie Clay Clemens (2007, S. 222) es umschreibt - „zwei Schritte vorwärts und einen Schritt zurück machte", geriet die SPD auch von anderer Seite unter Druck: Auf der linken Seite des Parteienspektrums hatte im Juli 2007 die neue Partei „Die Linke“ ihren Gründungsparteitag, womit sich die Linkspartei (PDS) und die WASG (Arbeit und soziale Gerechtigkeit - Die Wahlalternative) zu einer Kraft formierten und ein Bündnis schufen, das möglicherweise in Deutschland langfristig ein Fünf-Parteien-System etabliert.

Die Sozialdemokraten ließen mit ihrer Antwort nicht lange auf sich warten. Sie lancierten gegen den Willen der CDU das mit großem Aufwand im Koalitionsvertrag fixierte Thema der Mindestlöhne, was im Juni 2007 dann in einen Koalitionskompromiss mündete (zur Mindestlohndebatte umfassend Bispinck/Schulten 2008). In der Debatte um den Mindestlohn geht es jedoch nicht nur um diesen an sich, sondern, wie das Beispiel der Briefzustellung zeigt, auch um die Abwehr ausländischer Konkurrenz im Zuge der Liberalisierung der öffentlichen Infrastruktur.

Der Kabinettsbeschluss vom 8. April 2008, die Renten zum 1. Juli 2008 mittels Streckung der „Riestertreppe“ in der Anpassungsformel um zwei Jahre um $1,1 \% \mathrm{zu}$ erhöhen - was dem Standardrentner $13 €$ im Jahr einbringt -, ist eine Rückwärtsreform, die nun von beiden Parteien getragen wurde. Mit der Rentenerhöhung wurde im sozialpolitischen Wettlauf der beiden Parteien zunächst ein Gleichstand hergestellt. Bis Herbst 2009 wird dies in einen Stillstand münden. Es sei denn, das erneute Überholmanöver Jürgen Rüttgers, langjährigen Versicherten, die nur geringe Rentenbeiträge gezahlt haben, die Renten zu erhöhen, führt zu einem erneuten Wettlauf zwischen den beiden großen Parteien. Dabei ist jedoch zur Kenntnis zu nehmen, dass Rüttgers sich auch bei dieser Initiative auf den besagten Parteitag der CDU in Leipzig 2003 berufen kann. Denn auf diesem wurde beschlossen, dass die „Akzeptanz unseres Rentensystems [...] auf Dauer gefährdet“ sei, „wenn Versicherte trotz sehr langer Beitragszeiten lediglich Renten erhalten, die in der Höhe sehr nahe bei der Grundsicherung aus der Sozialhilfe oder sogar darunter liegen“" (zit. nach FAZ vom 23.04. 2008, S. 1).
Bei der sozialpolitischen Bilanz der Großen Koalition sollte bedacht werden, dass zwischen März 2006 und Februar 2008 in mehr als der Hälfte der Bundesländer Landtagswahlen stattfanden; bis zu den Bundestagswahlen im September 2009 werden mit Thüringen (Sommer 2008) und Bayern (September 2008) noch zwei weitere folgen und parallel zum Bund wird im Herbst 2009 auch noch in Sachsen, Brandenburg und im Saarland gewählt. Zählt man alle Wahlen zusammen, so werden wir dann eine Legislaturperiode des Bundestages mit elf bis 14 Landtagswahlen erlebt haben. Sozialpolitische Positionierungen sind nicht ganz unabhängig von Landtagswahlen; so sollte die Mindestlohndebatte zu einem Machtwechsel in Niedersachsen beitragen, was jedoch nicht gelang.

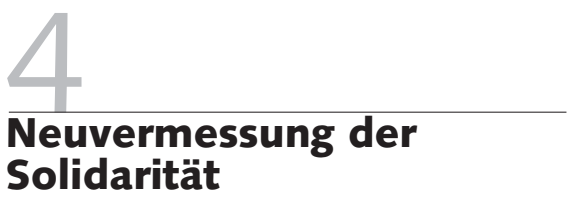

Das zweite Moment, das der Strukturwandel mit sich bringt, benenne ich als „Neuvermessung von Solidarität“. Solidarität beruht auf der Bereitschaft, sich innerhalb einer Gruppe, eines Sozialgefüges, gegenseitige Unterstützung und Hilfe zu geben und Opfer für das Wohlergehen der Gruppenmitglieder zu bringen (Beckert et al. 2004, S. 9). Wie viel Solidarität es in einer Gesellschaft gibt, wird maßgeblich von zwei Faktoren bestimmt: Zum einen vom Markteinkommen, damit aber auch von unterschiedlichen Marktlagen der Gesellschaftsmitglieder und damit verbundenen politökonomischen Verteilungskonflikten zwischen verschiedenen gesellschaftlichen Gruppen. Zum anderen wird Solidarität von der sozialen Konstitution jener Gruppen bestimmt, die, um solidarisch zu sein, ihre Ressourcen verteilen und Risiken unter ihren Mitgliedern poolen. Sowohl in den politökonomischen Konflikten als auch in der sozialen Konstitution von Gruppen sind in den letzten Jahren Veränderungen erkennbar. Ein Beispiel für den ersten Aspekt ist die Diskussion über die Frage, ob der intergenerationelle Solidaritätsvertrag, der 1957 mit der Einführung der Dynamisierung und des Umlageverfahrens in der Rentenversicherung begründet wurde, noch seine Gültigkeit besitzt oder ob vielmehr ein Konflikt zwischen Al- ten und Jungen eine neue Definition dessen verlangt, was intergenerationale Solidarität ist. Ein Beispiel dafür, wie Solidarität aufgrund der veränderten sozialen Konstitution von Gruppen neu vermessen wird, ist die tarifliche Sozialpolitik. Sie definiert Solidarität nicht mehr territorial, sondern funktional. Doch ich komme zunächst zur Frage der Neuvermessung von Solidarität zwischen Generationen.

\subsection{INTERGENERATIONALE VERSCHIEBUNGEN}

Wolfgang Streeck (2007, S. 279) fragt, ob wir uns „vom Generationenvertrag zum Generationenkonflikt" bewegen und Alterseffekte in der Politik heute eine größere Bedeutung besitzen. Er kommt dabei zu dem Schluss, dass der Konflikt zwischen Alt und Jung und seine Wirkung auf die Politik oftmals übertrieben dargestellt werden (ähnlich Goerres 2008, S. 11). Streeck verweist dabei darauf, dass eine mögliche „politische Machtergreifung der Älteren “ mit drei Entwicklungen zu tun habe (ebd., S. 282): mit der Überalterung, dem allgemeinen Wahlrecht und dem modernen Wohlfahrtsstaat: „Die gestiegene Lebenserwartung und die gesunkene Geburtenrate haben die Zahl der älteren Menschen und ihren Anteil an der Bevölkerung dramatisch steigen lassen; das allgemeine Wahlrecht macht die wachsende Bevölkerungsgruppe der Alten zu einem politischen Machtfaktor; und der moderne Wohlfahrtsstaat kann als Umverteilungsinstrument zugunsten von Wählergruppen genutzt werden, die ihre wirtschaftlichen Interessen am freien Markt nicht befriedigen können oder wollen“ (ebd., S. 283).

Ist die Rentenerhöhung, die die Große Koalition jüngst beschlossen hat, Ausdruck einer Seniorisierung der Sozialpolitik? Ist die vom CSU-Bundestagsabgeordneten Jens Spahn geäußerte Kritik an der Rentenerhöhung- „Den Rentnern von heute mag es helfen. Aber die junge Generation kostet das eine Menge Geld“ (zit. nach Tagesspiegel vom 17.03.2008, S. 4) - Anzeichen dafür, dass die Jungen den Generationenvertrag nicht mehr tragen wollen? Und steht die Replik des „Rotzlöffels“ für einen neuen und scharfen Ton zwischen Jung und Alt? Verschiedene aktuelle politikwissenschaftliche Abhandlungen widersprechen der These, dass es einen „Alt-JungKonflikt" gibt. So argumentiert Achim Goerres (2007, S. 3) in seiner Studie über 
den Einfluss des Alters auf die Ergebnisse der Bundestagswahlen, dass es in Deutschland bei den Wahlen „keinen Gegensatz zwischen Jung und Alt" gäbe und dass „Alter" keine politische Konfliktlinie im Parteienwettbewerb konstituiere. Er zeigt dabei auch, dass der Generationeneffekt das Wahlverhalten stärker beeinflusst als der Alterseffekt. ${ }^{1} \mathrm{Zu}$ bedenken ist auch, dass Rentnerinteressen heterogen sind und vom Anteil der Rente am Gesamteinkommen abhängen (Goerres 2008, S. 11). Zu einem ähnlichen Ergebnis gelangen Busemeyer et al. (2008) in ihrer international vergleichenden Studie zu sozialpolitischen Einstellungen von Rentnern. Dennoch sprach der ehemalige Bundespräsident Roman Herzog Anfang April 2008 polarisierend von einer „Rentnerdemokratie“. 2 Eine Umfrage, welche nach der Debatte über Herzogs Äußerung von Infratest dimap durchgeführt wurde, ergab jedoch keinen eindeutigen Alters- bzw. Rentnereffekt. So würden $44 \%$ der Rentner zugunsten der jüngeren Generation auf eine Rentenerhöhung verzichten, $52 \%$ allerdings nicht (Spiegel Online 2008).

Nach weiteren Strukturdaten scheinen die Alten im Parteien- und Verbändesystem eindeutig an Gewicht gewonnen zu haben (zum Folgenden vgl. Der Spiegel vom 07. 04.2008, S. 20): Die SeniorenUnion der CDU weist 58.000 Mitglieder auf - die Gesamtmitgliederzahl der CDU beträgt 532851 (per Ende März 2008) (abendblatt.de. 2008). Bei der Bundestagswahl 2005 erzielte die Union mit $42 \%$ bei den Rentnern ihr schlechtestes Wahlergebnis seit 1953, in der CDU stieg zwischen 1990 und 1997 der Anteil der über 60-jährigen Mitglieder von $29 \%$ auf $48 \%$, in der SPD von $25 \%$ auf $47 \%$, und der Sozialverband VdK, der sich unter seinem Präsidenten Walter Hirrlinger vehement für die Interessen der Rentner einsetzt, zählt inzwischen 1,4 Mio. Mitglieder. Jedoch gilt es hier, Streecks Einlassung (2007, S. 300) zu bedenken, dass die „Überalterung“der Parteimitglieder nicht unbedingt dazu führen muss, dass sich die Politik der Parteien radikal ändert. Parteien haben sich nämlich in den letzten Jahrzehnten auch von ihren Mitgliedern abgekoppelt. Parteienbindungen nehmen ab. Parteien lösen sich von ihrer Basis und entwickeln sich hin zu Kartellparteien. Auch auf Seiten der Gewerkschaften, so Streeck (2007, S. 296), könne man von einer „Herrschaft der Rentner“ nicht sprechen, auch wenn zwischen 1970 und 2000 der Anteil der Rentner an den Gewerkschaftsmitgliedern von $15,1 \%$ auf 19,0 \% gestiegen ist (ebd., S. 294). Angesichts dessen, dass es in der alternden Gesellschaft „zu einer politischen Polarisierung kommen" könne, folgert Streeck (2007, S. 302f.) dennoch, wenn auch mit der ihm eigenen Zurückhaltung gegenüber sozialwissenschaftlichen Befunden und Antworten, dass sich die Politik quasi präventiv durchaus der „Neuverhandlung des Generationenvertrages als Interessenausgleich zwischen Jung und Alt" widmen sollte. Wenn der Generationenvertrag derzeit zwar noch nicht neu verhandelt wird, so stünde er doch zunehmend in der Diskussion.

\subsection{VERÄNDERUNGEN INNERHALB DER SOLIDARITÄTSGRUPPEN}

Es gibt aber einen weiteren Faktor, der Solidarität neu vermisst. Dies ist der konstatierbare Trend, auf der Grundlage von tariflicher Sozialpolitik die territorial definierte Solidarität, auf der staatliche Sozialpolitik fußt, durch eine funktionale Solidarität zu ergänzen. Ich komme damit zur Neuvermessung von Solidarität aufgrund einer Veränderung der sozialen Konstitution von solidarischen Gruppen.

In Anlehnung an Thomas Marshalls Differenzierung zwischen politischen, sozialen und industriellen Bürgerrechten (Marshall 1964) lassen sich hinsichtlich der Konstitution von Solidargruppen idealtypisch zwei Modelle unterscheiden (zum Folgenden vgl. Trampusch 2008):

(1) Das „territoriale Modell“: Es zeichnet sich dadurch aus, dass auf der Grundlage von politischen Bürgerrechten durch die Einführung einer verpflichtenden Sozialversicherung oder von staatlichen Fürsorgeleistungen soziale Bürgerrechte geschaffen werden. Dies konstituiert Ansprüche an den Staat, der die Umverteilung organisiert und die Ressourcen verwaltet und verteilt.

(2) Das ,funktionale Modell“: Hier wird auf der Basis von industriellen Bürgerrechten umverteilt, indem Gewerkschaften sozialen Fortschritt mit Hilfe von in Tarifverträgen vereinbarten sozialen Leistungen durchsetzen. Gewerkschaften, so Marshall (1964, S. 94), können durch Tarifverträge „ein sekundäres System von Bürgerrechten “- eben industrielle Bürgerrechte - etablieren, das „parallel und ergänzend zum
System „politischer Bürgerrechte“ steht. In Deutschland hat in den letzten Jahren aufgrund der Tarifverträge zur Altersteilzeit und Altersvorsorge das funktionale Modell an Bedeutung gewonnen, insbesondere in der Rentenpolitik.

Tarifliche Altersvorsorge wurde in Deutschland im Rahmen der Riesterschen Rentenreform in einem breiten Maßstab eingeführt. Damit wurde sie parallel zu Kürzungen in der staatlichen Rentenversicherung und zu einer Ausweitung marktförmig organisierter Altersvorsorge etabliert. Während des Reformprozesses trat die IG BCE sehr stark als Befürworterin einer staatlichen Förderung von tariflicher Entgeltumwandlung auf. In den Tarifrunden 2000 und 2001 entwickelte sich sodann eine tarifpolitische Dynamik, die die IG Metall aufgrund früher Abschlüsse der Chemiegewerkschaft unter Druck setzte, auch ihre Tarifverträge für die Altersteilzeit und die Entgeltumwandlung zu öffnen.

Den Pfad tariflich gebundener Sozialpolitik baut die Chemiebranche derzeit weiter aus. Die IG BCE hat im April 2008 einen Tarifvertrag „Lebensarbeitszeit und Demografie“ vereinbart, der das Auslaufen der gesetzlichen Regelungen zur Altersteilzeit abfangen soll (IG BCE 2008). So werden ab 2009 betriebliche „Demografiefonds" eingerichtet, in die die Arbeitgeber pro Jahr für jeden Arbeitnehmer jeweils $300 €$ als „Demografiebeitrag“ einzahlen, wobei dieser Beitrag dynamisiert ist. $\mathrm{Ab}$ 2010 sollen dann aus diesen Fonds betriebsspezifische, vorzeitige und flexible Austritte aus dem Erwerbsleben finanziert werden. Diese Regelung kann von Beschäftigten im Alter zwischen 59 und 65 Jahren in Anspruch genommen werden. Sie verschiebt somit die Altersgrenze der alten Al-

\footnotetext{
Ein Alterseffekt würde sich zeigen, wenn es eine eindeutige Korrelation zwischen Wahlverhalten und der Zugehörigkeit zu einer bestimmten Altersgruppe bzw. Alterstufe geben würde. Dagegen weist der Generationeneffekt den Einfluss prägender gemeinsamer Erfahrungen - insbesondere bestimmter historischer Epochen oder Ereignisse auf das Verhalten bzw. die Einstellungen einer Gruppe aus.

2 Die Rentenerhöhung kommentierend, sagte $\mathrm{Her}$ zog der Bildzeitung: „Ich fürchte, wir sehen gerade die Vorboten einer Rentnerdemokratie: Die Älteren werden immer mehr, und alle Parteien nehmen überproportional Rücksicht auf sie. Das könnte am Ende in die Richtung gehen, dass die Älteren die Jüngeren ausplündern“ (n-tv.de 2008).
} 
tersteilzeitregelung (55 Jahre). Die Fonds wie auch die vorzeitige Teilrente sind sowohl mit den Regelungen zu Langzeitkonten verknüpft als auch mit dem Pensionsfonds der Chemieindustrie und den Regelungen zur tariflichen Altersvorsorge. Eine Verbindung wurde ferner auch zu einer Berufsunfähigkeitsversicherung geschaffen. Der neue Tarifvertrag sanktioniert und baut den Weg aus, auf dem sich die Chemiebranche bereits seit Mitte der 1970er Jahre befand und den sie seit den Sozialreformen Mitte der 1990er Jahre sukzessive erweitert hat: Es wird in dieser Branche gerade ein eigenes Sozialwerk geschaffen, das die staatlichen Sozialkürzungen zumindest partiell zu kompensieren versucht. Wieder hat das Chemiemodell Vorbildcharakter und findet Nachahmer. So hat auch die IG Metall die Altersteilzeit in die Tarifrunde 2008 eingebracht.

Tarifliche Sozialpolitik kann als ein Umverteilungsmodell interpretiert werden, das zwar weniger Solidarität und Umverteilung als staatliche Sozialpolitik offeriert, aber immer noch mehr als rein marktliche Lösungen. In einem System tariflicher Sozialpolitik basiert Umverteilung nämlich nicht mehr auf dem territorialen Prinzip, in dem mithilfe nationaler Fonds zwischen Sektoren, Berufen und Firmen Risikoausgleich betrieben wird, sondern auf dem funktionalen Prinzip. Mit Streeck (1999, S. 6f.) kann man festhalten, dass das funktionale Modell auf „kompetitiver und produktivistischer Solidarität" beruht. Gemäß diesem Prinzip bestimmt in erster Linie die Produktivität der tarifgebunde- nen Firmen den Grad der Umverteilung, was freilich nicht ausschließt, dass kollektivvertragliche oder rechtliche Regelungen das Umverteilungspotenzial erhöhen können. Die IG BCE fordert so auch, dass die Aufstockungsbeträge aus den Fonds, die den Rentenabschlag kompensieren sollen, steuer- und abgabefrei bleiben (IG BCE 2008).

\section{$(2)$ \\ Schlussfolgerung}

Die Frage steht im Raum: Wie wird es nach 2009 weitergehen? Nun: Politologen sind keine Propheten und ex-post meist sehr viel schlauer. Dennoch kann man zumindest eine Prognose wagen: Nur von einer „kleinen Koalition“ können wieder große Reformen erwartet werden und womöglich eine Rückwärtsreform der Rückwärtsreformen. Besonderer Handlungsbedarf wird in den Bereichen Rente, Pflege und Krankenversicherung entstehen, denn die wahlund parteipolitischen Geschenke des Jahres 2008 ziehen in naher Zukunft die Notwendigkeit von Leistungskürzungen oder einer weiteren Beitragserhöhung oder gar einer weiteren Mehrwertsteuererhöhung nach sich. Aus der Perspektive der neuen Koalition wäre die Rückwärtsreform der Rückwärtsreform dann wieder eine Vorwärtsreform. Um das Spiel nicht endlos zu treiben, kann man sich auch kurz fassen. Es gilt Aristoteles' Vermutung, dass „wir das Wesen jeglicher Erscheinung erst dann erken- nen, wenn sie ihren Höhepunkt erreicht und überschritten hat" (zit. nach Polanyi 1997[1944], S. 11).

Ist es für den deutschen Sozialstaat und seine Bewohner nun gut, wenn Solidarität neu vermessen wird? Blickt man in erfolgreiche Reformländer - wie die Niederlande oder Dänemark - so lautet die Antwort: Ja. Hier ist der Umbau des Sozialstaates nämlich nicht allein Sache partei- und wahlpolitischer Kalküle. Die Verbände übernehmen vielmehr auch in der Tarifpolitik Verantwortung, weil sie Tarifverträge zu sozial- und arbeitsmarktpolitischen Leistungen abschließen, was der Staat durch Maßnahmen im Sozial-, Arbeits- und Steuerrecht flankiert (vgl. hierzu auch Schmid in diesem Heft). In Deutschland hat der Bismarcksche Sozialstaat solche alten Formen vorstaatlicher Solidarität zur Seite gedrängt und lange Zeit völlig absorbiert. Die Niederlande und Dänemark zeigen jedoch, dass das Tarifvertragssystem beim Umbau der Sozialstaatlichkeit als institutionelle Flexibilitätsressource fungieren kann. In den Niederlanden sind im Übrigen auch die Senioren an der Verwaltung der Branchenpensionsfonds beteiligt. Die Fähigkeit der Verbände zur sektoralen Selbstorganisation sollte daher auch von der deutschen Politik nicht noch weiter geschwächt, sondern im Gegenteil gestärkt werden. Man kann es auch anders formulieren: Für den deutschen Sozialstaat muss das Verhältnis zwischen Staat und Verbänden neu justiert werden. Postkorporatismus ist nicht mit Entkorporatisierung gleichzusetzen. 
abendblatt.de (2008): CDU wird bald mehr Mitglieder haben als SPD (http://www.abendblatt.de/daten/2008/04/19/871149.html) Beckert, J./Eckert, J./Kohli, M./Streeck, W. (2004): Einleitung, in: Beckert, J./Eckert, J./Kohli, M./Streeck, W. (Hrsg.): Transnationale Solidarität: Chancen und Grenzen, Frankfurt/Main, New York, S. 9-14 Bispinck, R./Schulten, T. (2008): Aktuelle Mindestlohndebatte: Branchenlösung oder gesetzlicher Mindestlohn?, in: WSI-Mitteilungen 3, S. $151-158$

Busemeyer, M. R./Goerres, A./Welsche, S. (2008): Demands for Redistributive Policies in an Era of Demographic Aging. The Rival Pressures from Age and Class in 15 OECD Countries (MPIfG Discussion Paper 08/3), Köln

Clemens, C. (2007): Two Steps Forward, One Step Back: Merkel's CDU/CSU and the Politics of Welfare State Reform, in: German Politics 2, S. $222-246$

Goerres, A. (2007): Can We Reform the Welfare State in Times of "Grey" Majorities? The Myth of an Electoral Opposition between Younger and Older Voters in Germany (MPIfG Working Paper 07/5), Köln

Goerres, A. (2008): Populistische Rentenpolitik, in: Die Tageszeitung vom 28.04., S. 11

Industriegewerkschaft Bergbau Chemie Energie (IG BCE) (2008): Kräftige Entgelterhöhung in zwei Stufen, Medienmitteilung vom 16.04. (http://www.igbce.de/portal/site/igbce/menuitem.1aea7ff2eb76191 e9c5d3082c5bf21ca/)

Katzenstein, P. J. (1987): Policy and Politics in West Germany: The Growth of the Semi Sovereign State, Philadelphia

Lamping, W./Rüb, F. W. (2004): From the Conservative Welfare State to an 'Uncertain Something Else': German Pension Politics in Comparative Perspective, in: Policy \& Politics 2, S. 169-191

Lehmbruch, G. (2000): Institutionelle Schranken einer ausgehandelten Reform des Wohlfahrtsstaates: Das Bündnis für Arbeit und seine Erfolgsbedingungen, in: Czada, R./Wollmann, H. (Hrsg.): Von der Bonner zur Berliner Republik: 10 Jahre Deutsche Einheit, Leviathan, Sonderheft 19, Opladen, S. 89-112

Marshall, T. H. (1964): Class, Citizenship, and Social Development. Garden City, New York

Nullmeier, F. (2002): Auf dem Weg zu Wohlfahrtsmärkten?, in: Süß, W. (Hrsg.): Deutschland in den 90er Jahren: Politik und Gesellschaft zwischen Wiedervereinigung und Globalisierung, Opladen, S. 269-284 n-tv.de (2008): "Rentnerdemokratie." Alte "plündern“ Junge aus (http://www.n-tv.de/947264.html); alternativ: stern.de http://www. stern.de/politik/deutschland/:Rentenerh\%F6hung-HerzogRentnerdemokratie-/616949.html?nv=rss

Polanyi, K. (1997[1944]): The Great Transformation: Politische und öko- nomische Ursprünge von Gesellschaften und Wirtschaftssystemen, Frankfurt/Main

Schmidt, M. G. (2005): Sozialpolitik in Deutschland: Historische Entwicklung und internationaler Vergleich, Lehrbuch Grundwissen Politik 2, Wiesbaden

Schmidt, M. G. (2007): Die Sozialpolitik der zweiten rot-grünen Koalition (2002-2005), in: Egle, C./Zohlnhöfer, R. (Hrsg.): Das Ende des rot-grünen Projekts. Eine Bilanz der Regierung Schröder 2002-2005, Wiesbaden, S. $295-312$

Spiegel Online (2008): Fast die Hälfte der Rentner würde für Jüngere verzichten (http://www.spiegel.de/wirtschaft/0,1518,549561,00.html) Standfest, E. (1977): Soziale Selbstverwaltung: Zum Problem der Partizipation in der Sozialpolitik, in: Ferber, C. von/Kaufmann F.-X. (Hrsg.): Soziologie der Sozialpolitik, Kölner Zeitschrift für Soziologie und Sozialpsychologie, Sonderheft 19, Opladen, S. 424-437

Streeck, W. (1999): Competitive Solidarity: Rethinking the "European Social Model", MPIfG Working Paper 99/8, Köln

Streeck, W. (2005): Nach dem Korporatismus: Neue Eliten, neue Konflikte, MPIfG Working Paper 05/4, Köln

Streeck, W. (2007): Politik in einer alternden Gesellschaft. Vom Generationenvertrag zum Generationenkonflikt?, in: Gruss, P. (Hrsg.): Die Zukunft des Alterns: Die Antwort der Wissenschaft, München, S. 279-304 Streeck, W. (2008): Re-Forming Capitalism. The Slow Transformation of the German Political Economy, Oxford

Streeck, W./Trampusch, C. (2005): Economic Reform and the Political Economy of the German Welfare State, in: German Politics 2, S. 174-195 Trampusch, C. (2006a): Status quo vadis? Die Pluralisierung und Liberalisierung der "Social-Politik" als Herausforderung für die politikwissenschaftliche und soziologische Sozialpolitikforschung, in: Zeitschrift für Sozialreform 3, S. 299-323

Trampusch, C. (2006b): Postkorporatismus in der Sozialpolitik - Folgen für Gewerkschaften, in: WSI-Mitteilungen 6, S. 347-352

Trampusch, C. (2008): Warum und wie Industrielle Beziehungen den Parteieneffekt auf wohlfahrtsstaatliche Retrenchmentpolitik beeinflussen Ein Vergleich zwischen den Niederlanden, Dänemark, Deutschland und Frankreich, in: Zeitschrift für Vergleichende Politikwissenschaft 2, im Erscheinen

Winter, T. von (1997): Sozialpolitische Interessen: Konstituierung, politische Repräsentation und Beteiligung an Entscheidungsprozessen, BadenBaden 